\title{
Neutrino SuperBeams in Europe
}

\author{
Mauro Mezzetto ${ }^{\mathrm{a}}$

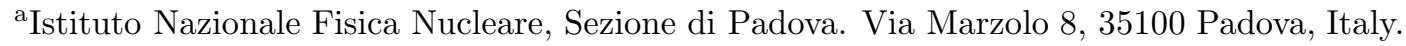

A brief report of the developments in Europe towards next generation neutrino oscillation long baseline experiments capable of measuring the still unknown parameters $\theta_{13}$ and $\delta_{\mathrm{CP}}$.

\section{Introduction}

Three parameters are still unknown in neutrino oscillations: the mixing angle $\theta_{13}$, the $\mathrm{CP}$ phase $\delta_{\mathrm{CP}}$ and the neutrino mass hierarchy parametrized by $\operatorname{sign}\left(\delta m_{23}^{2}\right)$. They can be detected in a long baseline neutrino experiment looking for sub-leading $\nu_{\mu} \rightarrow \nu_{e}$ oscillations.

The first experiment optimized to those searches will be T2K [1], at the J-Parc $50 \mathrm{GeV}$, 0.75 MW proton synchrotron, scheduled to start in 2009, with a $\theta_{13}$ sensitivity 20 times better than the present experimental limit [2]. It could be complemented by the No $\nu$ a [3] experiment in the NuMi beam line and by the reactor experiment Double Chooz [4]. Even the combination of those three experiments however will not have any sensitivity to $\delta_{\mathrm{CP}}[5]$.

A new generation of experiments has to be designed to address the search of leptonic CP violation. It will be characterized by neutrino SuperBeams and/or Beta Beams.

Neutrino factories are designed to be the ultimate facility for neutrino oscillation searches, [6]. They will probably be ready in a longer timescale and they will not be discussed in this report.

In this paper are illustrated the studies done in Europe to address the needs of these future long baseline experiments. Most of these developments have been discussed inside the "Neutrino Oscillation Working Group" [7], a forum of discussion promoted by ECFA and recently endorsed by the european network BENE [8].

\section{Sub-Leading $\nu_{\mu} \rightarrow \nu_{e}$ oscillations}

The parameters $\theta_{13}, \delta_{\mathrm{CP}}$ and $\operatorname{sign}\left(\delta m_{23}^{2}\right)$ can be extracted by measuring sub-leading $\nu_{\mu} \rightarrow \nu_{e}$ oscillations. For a parametrization of this probability see reference [9]. $\nu_{\mu} \rightarrow \nu_{e}$ oscillations have a leading term driven by the solar parameters, but at the baseline defined by $\delta m_{23}^{2}, L_{a t m}$, they are driven by $\theta_{13}$ terms for $\sin ^{2} 2 \theta_{13}>10^{-3}$.

$\theta_{13}$ searches look for experimental evidence that $P_{\nu_{\mu} \rightarrow \nu_{e}}>P_{\nu_{\mu} \rightarrow \nu_{e}}\left(\theta_{13}=0\right)$ while leptonic $\mathrm{CP}$ violation searches look for $A_{C P}=$ $\frac{P\left(\nu_{\mu} \rightarrow \nu_{e}\right)-P\left(\bar{\nu}_{\mu} \rightarrow \bar{\nu}_{e}\right)}{P\left(\nu_{\mu} \rightarrow \nu_{e}\right)+P\left(\bar{\nu}_{\mu} \rightarrow \bar{\nu}_{e}\right)} \neq 0$. This asymmetry results to be proportional to $1 / \sin \theta_{13}$ while $P_{\nu_{\mu} \rightarrow \nu_{e}}$ is proportional to $\sin ^{2} 2 \theta_{13}$, at the first order $A_{C P}$ sensitivity is independent from $\theta_{13}$, provided that $\theta_{13}$ is big enough to allow for a sizable $\nu_{e}\left(\bar{\nu}_{e}\right)$ signal. Matter effects also produce differences from $P_{\nu_{\mu} \rightarrow \nu_{e}}$ and $P_{\bar{\nu}_{\mu} \rightarrow \bar{\nu}_{e}}$, they are proportional to the baseline and at $100 \mathrm{~km}$ they result to be negligible.

The richness of $\nu_{\mu} \rightarrow \nu_{e}$ transitions is also their weakness: it will be very difficult for pioneering experiments to extract the single values of all the parameters. Correlations are present between $\theta_{13}$ and $\delta_{\mathrm{CP}}$ and clone solutions exist in absence of information about $\operatorname{sign}\left(\delta m_{23}^{2}\right)$ and about the $\pi / 2-\theta_{23}$ ambiguity [10].

\section{Neutrino SuperBeams}

SuperBeams could be defined as conventional neutrino beams generated by a proton driver with more than $1 \mathrm{MW}$. At that power no conventional neutrino target could survive, so they will require R\&D for the target design [11].

Pushed to those intensities conventional neutrino beams will hit their ultimate limitations, namely the presence of 4 neutrino flavors in the neutrino beam $\left(\nu_{e}, \bar{\nu}_{\mu}, \bar{\nu}_{e}\right.$ besides the main neutrino component, $\left.\nu_{\mu}\right)$ and the difficulty to pre- 
cisely predict fluxes and contaminations because of the lack of knowledge of secondary particle production cross sections.

SuperBeam experiments have been proposed in the States [3], [12], and in Japan [1]. This latter project, T2K phase II, probably the most powerful proposal, plans a machine upgrade from 0.75 MW to 4 MW fired to HyperKamiokande, a detector with a fiducial volume 24 times bigger than SuperKamiokande. Its physics potential has been discussed in [13] showing that $\delta_{\mathrm{CP}}$ can be detected at $3 \sigma$ if bigger than $20^{\circ}$ and if $\theta_{13}$ is bigger than $2^{\circ}$. This $\theta_{13}$ range corresponds to the T2K phase I sensitivity. Sensitivity depends very much from the systematic errors level, systematic errors bigger than $2 \%$ would significantly degrade the experimental sensitivity.

\subsection{Extensions of CNGS}

CNGS neutrino beam has been optimized for $\nu_{\tau}$ appearance, has a mean neutrino energy of $17 \mathrm{GeV}$ and detectors at a baseline of $732 \mathrm{~km}$ (CERN-LNGS). This optimization is very different from a $\theta_{13}$ optimization, where for the same baseline the mean neutrino energy should be $\sim 1.5 \mathrm{GeV}$. Two studies have been done to re-use part of the existing infrastructure to build a neutrino beam optimized for $\theta_{13}$ searches. The study of reference [14] considers a new beam optics capable to produce a neutrino beam of 1.5 $\mathrm{GeV}$ mean energy fired to a 3 kton Icarus detector at Gran Sasso. The experiment would reach $1 / 3$ of the sensitivity of T2K phase I in a 5 years run at the nominal CNGS intensity.

A second study considered a low energy neutrino beam (1.5 GeV mean energy) fired to a detector made of $44 \mathrm{k}$ phototubes deployed 1000 $\mathrm{m}$ underwater, equipping 2 Mton of water, $2^{\circ}$ degrees off-axis, $1200 \mathrm{~km}$ from CERN (CNGT) [15]. In this case the detector would be placed at the second oscillation maximum and if movable it could take data both at the minimum and at the maximum of oscillation probability. Sensitivity would be marginally worse than T2K phase I, in a 5 years data taking [15].

These performances are similar to T2K phase I but the timescale would be significantly later: allowing for a 5 years run for the standard CNGS beam plus a couple of years for the beam modifications, these experiments could only start after the end of $\mathrm{T} 2 \mathrm{~K}$ data taking.

\subsection{Beams from a $20-50 \mathrm{GeV}$ high inten- sity synchrotron}

A rapid cycling synchrotron with proton energy in the $20-50 \mathrm{GeV}$ range, could be foreseen for the LHC luminosity upgrade. Such a machine would be very similar to the J-Parc $50 \mathrm{GeV}, 0.75 \mathrm{MW}$ proton synchrotron and could reach $4 \mathrm{MW}$.

Performances of a 3 kton liquid argon detector (with 100\% efficiency, no detector backgrounds and no systematic errors) exposed to a neutrino beam produced by a $20 \mathrm{GeV}, 6.5 \mathrm{MW}$ proton driver (PS++) have been published in [16]. $\theta_{13}$ sensitivity would be similar to T2K phase I. With the known technologies no significant improvement from these performances can be foreseen in terms of detector mass times efficiency by a neutrino detector inside a LNGS hall $\left(40000 \mathrm{~m}^{3}\right)$. Other experimental developments could overcome this limitation, such as high density calorimeters, surface detectors or new underground facilities. They haven't been studied yet.

\subsection{SPL SuperBeam at CERN}

The Super Proton Linac (SPL) is a proton driver designed to deliver $2 \mathrm{~mA}$ of $2.2 \mathrm{GeV}$ (kinetic energy) protons [17]. It could be the driver either of a Beta Beam or of a Neutrino Factory. Protons would be delivered to an accumulator, that could be hosted in the ISR tunnel, in order to have beam batches $23 \mathrm{~ns}$ long. Pions are produced by the interactions of the $2.2 \mathrm{GeV}$ proton beam with a liquid mercury target [11] and focused with magnetic horns [18]. The resulting neutrino flux [18] has a neutrino energy $\left\langle E_{\nu}\right\rangle=260 \mathrm{MeV}$ and the optimal baseline would be of about $100 \mathrm{~km}$.

There are several advantages by running at such small $\nu$ energies. Given the relatively short baseline, matter effects are negligible and don't compete with leptonic CP violating effects. Protons are below the kaon production threshold, reducing $\nu_{e}$ backgrounds and the incertitudes related to their estimation. $\pi^{\circ}$ rejection is favored thanks to the wide $\gamma$ s opening angle ${ }^{1}$. Charged

\footnotetext{
${ }^{1}$ Indeed better rejections than $\mathrm{T} 2 \mathrm{~K}$ (running at $\left\langle E_{\nu}\right\rangle \simeq$
} 
current (CC) events are for the largest part quasi elastics, the event category best reconstructed in a water Čerenkov detector.

On the other hands cross sections are small at these energies and change very rapidly with energy. Antineutrino interaction rates are suppressed either because antineutrino/neutrino cross sections ratio is at a minimum: $(\simeq 1 / 4)$ and because $\pi^{-}$hadroproduction is disfavored at $2.2 \mathrm{GeV},{ }^{2}$. Fermi motion prevents an accurate event energy reconstruction. Atmospheric neutrino backgrounds are severe because of the high flux below $0.5 \mathrm{GeV}$ and the limited rejection factor provided by the poor angular resolution; this is the reason why an accumulator is needed downstream the proton driver to keep the duty cycle low. Given the baseline, $\operatorname{sign}\left(\delta m_{23}^{2}\right)$ cannot be measured. It should be noted that $\mathrm{CC}$ rates generated by the SPL-SB at the optimal baseline, $41 \mathrm{CC}$ events/kton/year, are smaller than T2K phase I rates, $\sim 100$ events/kton/year, the driver of T2K (50 GeV) having 6 times less power.

SPL SuperBeam (SPL-SB) performances have been computed in [19] for a water Čerenkov detector, 440 kton fiducial (20 times SuperKamiokande) [20], $130 \mathrm{~km}$ from CERN, under the Frejus. Given the poor energy resolution a counting experiment has been evaluated, while it has been shown in a recent paper [21] that energy reconstruction may be used having beneficial effects. $\theta_{13}$ sensitivity is shown in Fig. 2, while $\delta_{\mathrm{CP}}$ discovery potential $(3 \sigma)$ is shown in Fig. 3. These plots don't take into account the $\operatorname{sign}\left(\delta m_{23}^{2}\right)$ and $\pi / 2-\theta_{23}$ ambiguities, for a computation of SPL-SB sensitivities having them included see reference [22].

SPL energy, $2.2 \mathrm{GeV}$, was originally fixed having in mind the re-usage of LEP RF cavities. More modern cavities could allow higher energies. In a recent paper [23], $\theta_{13}$ sensitivities as function of the proton beam energy, keeping fixed the power of the machine and the experimental baseline, have been computed. At an energy of

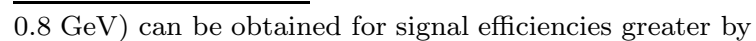
about a factor 2 .

${ }^{2}$ To compensate that, 2 years of $\nu_{\mu}$ and 8 years of $\bar{\nu}_{\mu}$ running must be planned for the leptonic CP violation searches.
3.5 GeV, and focusing higher momentum pions, most of the weak points of the SPL-SB are cured: hadroproduction becomes more favorable and an higher energy neutrino beam can be produced $\left(\left\langle E_{\mu}\right\rangle \simeq 350 \mathrm{MeV}\right)$ allowing for a moderate energy binning (200 MeV/bin). CC interaction rate would be raised from 41 to 122 events/kton/year, 3 . Performances in this new configuration (SPLSB $3.5 \mathrm{GeV}$ ) are shown in Fig. 2 and $3 .{ }^{4}$

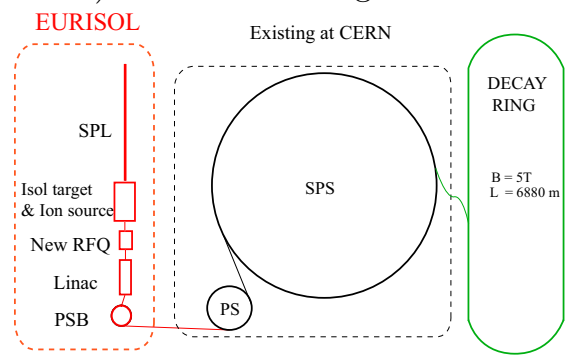

Figure 1. Schematic layout of the Beta Beam complex. At left, the low energy part is largely similar to the EURISOL project [29]. The central part (PS and SPS) uses existing facilities. At right, the decay ring has to be built.

\section{Beta Beams}

Beta Beams $(\beta \mathrm{B})$ have been introduced by $\mathrm{P}$. Zucchelli in 2001 [24]. The idea is to generate pure, well collimated and intense $\nu_{e}\left(\bar{\nu}_{e}\right)$ beams by producing, collecting, accelerating radioactive ions and storing them in a decay ring in $10 \mathrm{~ns}$ long bunches, to suppress the atmospheric neutrino backgrounds. This approach overcomes the limitations of conventional neutrino beams: $\beta B$ would be virtually background free and fluxes could be easily computed by the properties of the beta decay of the parent ion and by its Lorentz boost factor $\gamma$.

The best ion candidates so far are ${ }^{18} \mathrm{Ne}$ and ${ }^{6} \mathrm{He}$ for $\nu_{e}$ and $\bar{\nu}_{e}$ respectively. A baseline study for a Beta Beam complex has been produced at CERN [25] and shown schematically in Fig. 1. In this scenario Beta Beam neutrino energies are below $0.5 \mathrm{GeV}$ and the ideal technology would be again water Čerenkov.

\footnotetext{
This improvement includes a longer $(20 \mathrm{~m}$ to $40 \mathrm{~m})$ and wider (1m diameter to $2 \mathrm{~m}$ ) decay tunnel.

${ }^{4} \theta_{13}$ sensitivity of Fig. 2 is better than what quoted in [23] because it is computed taking into account the energy information.
} 
4

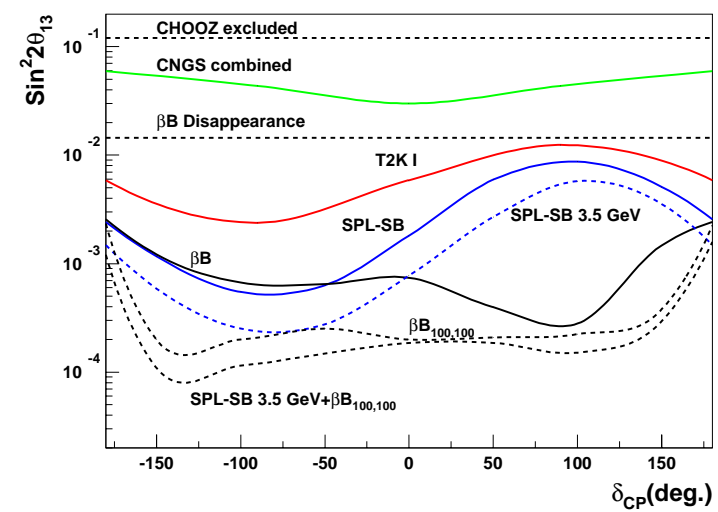

Figure 2. $\theta_{13} \quad 90 \% \mathrm{CL}$ sensitivity as function of $\delta_{\mathrm{CP}}$ for $\delta m_{23}^{2}=2.5 \cdot 10^{-3} \mathrm{eV}^{2}, \operatorname{sign}\left(\delta m_{23}^{2}\right)=1$, $2 \%$ systematic errors. CNGS and T2K curves are taken from [9], BNL from [12]. SPL-SB sensitivities have been computed for a 5 years $\nu_{\mu}$ run, $\beta \mathrm{B}$ for a 5 years $\nu_{e}+\bar{\nu}_{e}$ run.

The reference $\beta \mathrm{B}$ fluxes are $2.9 \cdot 10^{18}{ }^{6} \mathrm{He}$ useful decays/year and $1.1 \cdot 10^{18}{ }^{18} \mathrm{Ne}$ decays/year. The SPS could accelerate ${ }^{6} \mathrm{He}$ ions at a maximum $\gamma$ value of $\gamma_{6} \mathrm{He}=150$ and ${ }^{18} \mathrm{Ne}$ ions up to $\gamma_{18 \mathrm{Ne}}=250$. In the baseline scenario the two ions circulate in the decay ring at the same time. This is feasible provided that their $\gamma$ are in the ratio $\gamma_{6} \mathrm{He} / \gamma_{18} \mathrm{Ne}=3 / 5$. The same fluxes can be obtained by running the two ions separately [26]. This allows a better optimization of the physics potential of the machine [27].

The baseline scenario physics potential has been computed in [28] for $\gamma_{6} \mathrm{He}=60, \gamma_{18} \mathrm{Ne}=$ 100, see Fig. 2 and 3 . In the same plots performances computed with both ions at $\gamma=$ 100, exploiting energy shape information (200 $\mathrm{MeV} / \mathrm{bin})$, are also displayed $\left(\beta \mathrm{B}_{100,100}\right)$, with a clear gain in sensitivity. The overall optimization and the assesment of the physics potential of the baseline Beta Beam will be the argument of forthcoming papers. Sensitivities taking into account all the parameter degeneracies and ambiguities have been computed in [22].

$\beta \mathrm{B}$ and SPL-SB are perfectly compatible both in terms of SPL proton economics and in terms of optimal baseline. The same detector could then be exposed to $2 \times 2$ beams $\left(\nu_{\mu}\right.$ and $\bar{\nu}_{\mu} \times \nu_{e}$ and $\left.\bar{\nu}_{e}\right)$ having access to $\mathrm{CP}, \mathrm{T}$ and $\mathrm{CPT}$ searches in the same run. Physics potential of this combination of beams is illustrated in Fig. 2 and 3.

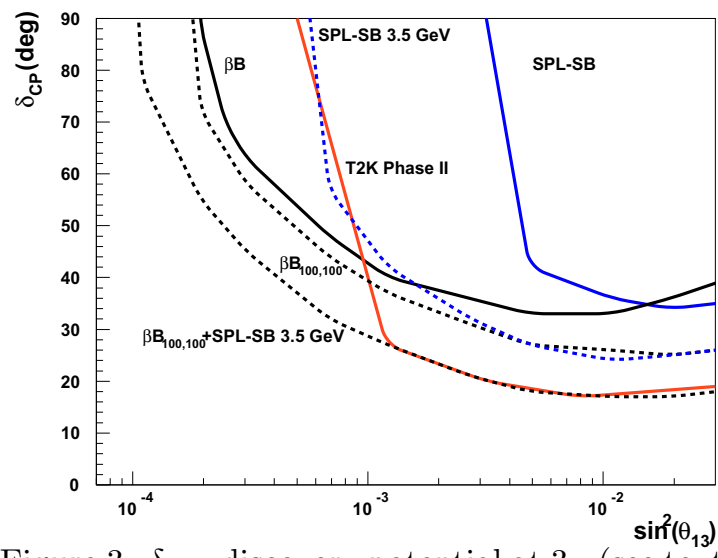

Figure $3 . \delta_{\mathrm{CP}}$ discovery potential at $3 \sigma$ (see text) computed for a 10 years run, $2 \%$ systematic errors. T2K phase II curve is taken from [13].

Beta Beam capabilities for ions accelerated at higher energies than those allowed by SPS have been computed in [30], assuming that the same ion fluxes of the baseline scenario can be maintained. In particular accelerating ${ }^{6} \mathrm{He}$ ions up to $\gamma=350$ and placing the megaton detector at about $700 \mathrm{~km}, \theta_{13}, \operatorname{sign}\left(\delta m_{23}^{2}\right)$ and $\delta_{\mathrm{CP}}$ sensitivities similar or better to those of a Neutrino Factory could be reached. Additional ideas about high energy Beta Beams have been published in [31]. For a review see also [27].

\section{Conclusions}

A summary of future long baseline experiments is in Tab. 1. The best candidates so far are Beta Beams, an approach virtually free from intrinsic backgrounds and systematics.

This project covers several important physics themes, ranging from the very deep synergies with EURISOL, aiming at producing high intensity radioactive beams for nuclear physics studies, to the excellent physics capabilities of the megaton water Čerenkov detector in its own [20].

Beta Beams could be complemented by a neutrino SuperBeam generated by the SPL 4MW, 2.2 $\mathrm{GeV}$ proton Linac fired to the same detector, allowing for $\mathrm{CP}$ and $\mathrm{T}$ violation searches as well as CPT violation in neutrino oscillations.

\section{REFERENCES}

1. Y. Itow et al., hep-ex/0106019. 
Table 1

Summary table of different LBL options. T2K phase II $\sin ^{2} 2 \theta_{13}$ sensitivity is extrapolated from T2K phase I. PS ++ numbers are normalized to $4 \mathrm{MW}$ power. Regarding $\beta \mathrm{B}$, the $\nu_{\mu}{ }^{C C}$ line indicates the sum of $\nu_{e}^{C C}$ and $\bar{\nu}_{e}^{C C}$ rates while the $\pi / \nu_{e}$ line indicates the fraction of NC backgrounds normalized to the non oscillated $\nu_{e}+\bar{\nu}_{e}$ rate.

\begin{tabular}{lc|ccccccc}
\hline & & $\mathrm{T} 2 \mathrm{~K}$ & $\mathrm{~T} 2 \mathrm{~K} 2$ & $\mathrm{PS}++$ & SPL & SPL 3.5 & $\beta \mathrm{B}$ & $\beta \mathrm{B}_{100,100}$ \\
\hline p-driver & $(\mathrm{MW})$ & 0.75 & 4 & 4 & 4 & 4 & 0.4 & 0.4 \\
p beam energy & $(\mathrm{GeV})$ & 50 & 50 & 20 & 2.2 & 3.5 & $1-2.2$ & $1-2.2$ \\
$\left\langle E\left(\nu_{\mu}\right)\right\rangle$ & $(\mathrm{GeV})$ & 0.7 & 0.7 & 1.6 & 0.27 & 0.35 & 0.3 & 0.4 \\
$L$ & $(\mathrm{Km})$ & 295 & 295 & 732 & 130 & 130 & 130 & 130 \\
Off-Axis & & $2^{0}$ & $2^{0}$ & - & - & - & - & - \\
$\nu_{\mu}^{C C}\left(\nu_{e}^{C C}\right)$ (no oscillation) & $(\mathrm{Kt} /$ year $)$ & 100 & 500 & 450 & 41 & 122 & 38 & 56 \\
$\nu_{e}^{C C} / \nu_{\mu}^{C C}$ & $(\%)$ & 0.4 & 0.4 & 1.2 & 0.4 & 0.7 & 0 & 0 \\
\hline Detector Fid. Mass & $(\mathrm{kton})$ & 22.5 & 540 & 2.2 & 440 & 440 & 440 & 440 \\
Material & & $\mathrm{H}_{2} \mathrm{O}$ & $\mathrm{LAr}$ & $\mathrm{H}_{2} \mathrm{O}$ & $\mathrm{H}_{2} \mathrm{O}$ & $\mathrm{H}_{2} \mathrm{O}$ & $\mathrm{H}_{2} \mathrm{O}$ & $\mathrm{H}_{2} \mathrm{O}$ \\
Signal efficiency & $(\%)$ & 40 & 40 & 100 & 70 & 70 & 60 & 70 \\
$\pi^{\circ} / \nu_{e}\left(\pi / \nu_{e}\right)$ & $(\%)$ & 80 & 80 & 0 & 30 & 30 & 0.2 & 0.2 \\
$\sin ^{2} 2 \theta_{13} \cdot 10^{4}$ & $(90 \% \mathrm{CL})$ & 60 & 6 & 64 & 18 & 7 & 7 & 2 \\
\hline
\end{tabular}

2. M. Apollonio et al., Eur. Phys. J. C 27 (2003) 331, [hep-ex/0301017].

3. I. Ambats et al. [NOvA Collaboration], FERMILAB-PROPOSAL-0929

4. F. Ardellier et al., hep-ex/0405032.

5. P. Huber et al., hep-ph/0403068. K. B. McConnel and M. H. Shaevitz, hep-ex/0409028.

6. S. Geer, Phys. Rev. D 57 (1998) 6989 [Erratum-ibid. D 59 (1999) 039903], [hepph/9712290]. M. Apollonio et al., hep$\mathrm{ph} / 0210192$.

7. Neutrino Oscillation Working Group, http://axpd24.pd.infn.it/nowg.

8. http://bene.na.infn.it/

9. P. Migliozzi and F. Terranova, Phys. Lett. B 563 (2003) 73, [hep-ph/0302274].

10. H. Minakata, H. Nunokawa and S. J. Parke, Phys. Rev. D 66 (2002) 093012 [hep$\mathrm{ph} / 0208163$ and references therein.

11. H.D.Haseroth et at., AIP Proceedings 721, 48-59, 2003. M.S.Zisman, AIP Proceedings 721, 60-67, 2003.

12. M. V. Diwan et al., Phys. Rev. D 68, 012002 (2003), [hep-ph/0303081].

13. T. Kobayashi, J. Phys. G 29 (2003) 1493.

14. A. Rubbia and P. Sala, JHEP 0209 (2002) 004 [hep-ph/0207084].

15. A.E.Ball et al., CERN-SPSC-2004-025,SPSCM-723, 21 September, 2004.

16. A. Ferrari, A. Rubbia, C. Rubbia and P. Sala, New J. Phys. 4 (2002) 88 [hep-ph/0208047].

17. B. Autin et al., CERN-2000-012

18. A. Blondel et al., CERN-NUFACT-53. S. Gilardoni et al., J. Phys. G 29 (2003) 1801.

19. J. J. Gomez-Cadenas et al., hep-ph/0105297. A. Blondel et al., Nucl. Instrum. Meth. A 503 (2001) 173. M. Mezzetto, J.Phys.G29:17711776, 2003; [hep-ex/0302005].

20. UNO Collaboration, hep-ex/0005046

21. A.Blondel et al., Nucl. Instrum. Meth. A 535 (2004) 665 .

22. A. Donini et al., hep-ph/0406132. A. Donini et al., hep-ph/0411402.

23. J. E. Campagne and A. Cazes, hepex/0411062.

24. P. Zucchelli, Phys. Lett. B 532 (2002) 166.

25. B. Autin et al., physics/0306106. M. Benedikt, S. Hancock and M. Lindroos, Proceedings of EPAC, 2004, http://accelconf.web.cern.ch/AccelConf/e04

26. Mats Lindroos, private comunication.

27. M. Mezzetto, hep-ex/0410083.

28. M. Mezzetto, J.Phys.G29:1781-1784, 2003; [hep-ex/0302007]. J. Bouchez, M. Lindroos and M. Mezzetto, AIP conference proceedings, Vol. 721, 37-47, 2003. [hep-ex/0310059].

29. http://www.ganil.fr/eurisol/

30. J. Burguet-Castell at al., Nucl. Phys. B 695 (2004) 217 [hep-ph/0312068].

31. F. Terranova et al., hep-ph/0405081. 